\title{
Parametric and nonparametric genome scan analyses for human handedness
}

\author{
Tom Van Agtmael ${ }^{\star, 1,2}$, Susan M Forrest ${ }^{1,2,3}$, Jurgen Del-Favero ${ }^{4}$, Christine Van Broeckhoven ${ }^{4}$ \\ and Robert Williamson ${ }^{1}$
}

\begin{abstract}
${ }^{1}$ Murdoch Childrens Research Institute, Royal Children's Hospital, Melbourne, Australia; ${ }^{2}$ Department of Paediatrics, The University of Melbourne, Melbourne, Victoria 3052, Australia; ${ }^{3}$ Australian Genome Research Facility, Walter and Eliza Hall Institute of Medical Research, Melbourne, Victoria 3052, Australia; ${ }^{4}$ Department of Molecular Genetics, Flanders Interuniversity Institute for Biotechnology (VIB), University of Antwerp (UIA), 2610 Antwerpen, Belgium
\end{abstract}

We have performed a genome scan using 25 nuclear families consisting of right-handed parents with at least two left-handed children. Handedness was assessed as a qualitative trait using a laterality quotient. Laterality quotients indicate the direction of handedness, which is hand preference for performing unimanual tasks. Both parametric and nonparametric linkage analyses were applied. The parametric analysis using the single-locus genetic model of Klar resulted in four different regions with LOD scores higher than 1. The region on chromosome 10q26 gave a suggestive LOD score of 2.02 at a recombination fraction of 0.05 . Nonparametric analysis gave an NPL score for this region of 2.16. However, further fine mapping of the region on chromosome 10q26 failed to obtain a higher LOD score. These results suggest that handedness is a human quantitative trait locus and that the proposed non-Mendelian monogenic models are incorrect.

European Journal of Human Genetics (2003) 11, 779-783. doi:10.1038/sj.ejhg.5201048

Keywords: handedness; linkage analysis; complex traits

\section{Introduction}

Left-handedness has a frequency of around $10 \%$ in the general population with a slightly higher frequency in the male population compared to the female population. ${ }^{1}$ The incidence of left-handedness decreases strikingly in the elderly to less than $5 \%$ above the age of $70 .{ }^{1}$ Both reduced survival fitness and the presence of social pressures against left-handedness in the beginning of the 20th century have been invoked to explain the declining incidence. ${ }^{2}$ Lefthandedness is highly correlated with cerebral asymmetry. Right cerebral dominance occurs in $4 \%$ of right-handed individuals compared to $27 \%$ of left-handed individuals. ${ }^{3}$

*Correspondence: Dr Van Agtmael, MRC Human Genetics Unit, Western General Hospital, Crewe Road, Edinburgh EH4 2XU, UK.

Tel: + 44131332 2471; Fax: + 44131467 8456;

E-mail: tom.vanagtmael@hgu.mrc.ac.uk

Received 28 January 2003; revised 16 April 2003; accepted 7 May 2003
The genetics of left-handedness is a highly debated issue and both environmental and genetic models have been proposed. However, twin, family and adoption studies support a genetic background to human handedness. ${ }^{4-7}$ Several proposed genetic models ${ }^{8-10}$ incorporate the biological feature of fluctuating asymmetry, which indicates that handedness is determined by chance due to the accumulation of stochastic events during embryonic development. ${ }^{9}$ Klar proposed a single-gene model in which a bi-allelic gene, RGHT, with alleles $\mathrm{R}$ and $\mathrm{r}$ determine handedness. Individuals who have the genotypes $\mathrm{R} / \mathrm{R}$ and $\mathrm{R} / \mathrm{r}$ will be right-handed, while the genotype $\mathrm{r} / \mathrm{r}$ results in fluctuating asymmetry and thus 50\% right-handedness and $50 \%$ left-handedness. ${ }^{10}$ Klar proposed gene frequencies of 58 and $42 \%$ for alleles $\mathrm{R}$ and $\mathrm{r}$, respectively. Other genetic models have been proposed, such as the Right Shift model $^{8}$ and the model by McManus. ${ }^{9}$ However, the exact genetic model of human handedness remains to be 
elucidated. Recently, family data on more than 2000 families suggested a X-linked inheritance. ${ }^{11}$

The different genetic models of Klar, Annett and McManus match some epidemiological data. ${ }^{6,8-10,12}$ For linkage analysis, the model of Klar is the more reductionist model compared to the Right Shift model, which invoked different thresholds for different subpopulations. ${ }^{8}$ The model of McManus is less reductionist due to the incorporation of a modifier locus on the X-chromosome. ${ }^{6}$

Nuclear families consisting of right-handed parents with at least two left-handed children are the most informative pedigree structure in linkage studies using the model of Klar as it maximises the proportion of parents that are heterozygous $\mathrm{R} / \mathrm{r}$ at the locus level. ${ }^{13}$ Consequently, and analogous to standard autosomal recessive disorders, the heterozygosity of the right-handed parents and the homozygosity of the left-handed children at the locus level will result in informative meioses and more power in linkage calculations. ${ }^{13}$ Power analysis showed that the nuclear families were only powerful for the model of Klar and not for the model of McManus. ${ }^{13}$

Candidate gene analysis of genes involved in left-right asymmetry using these families resulted in the exclusion of every candidate gene tested. ${ }^{14}$ This study expands on our candidate gene analysis by performing a $10 \mathrm{~cm}$ genome scan in order to map a locus for human handedness.

Using the single-locus model of Klar, simulation studies indicated that 25 families would be sufficient to map a locus for human handedness.

\section{Materials and methods Sample collection and DNA extraction}

The study was approved by the human research ethics committee of the Women and Children's Hospital, Melbourne, Australia. Families were recruited through a call for families in the Australian media, which included an article in a local newspaper. All families are from Caucasian origin and living in Australia. Samples were collected of 25 families consisting of at least two left-handed children from right-handed parents. A total of 127 individuals were used in the genome scan, of which 72 (56.7\%) individuals were right-handed and 55 (43.3\%) individuals were lefthanded.

Mouthwashes were used as the primary source of DNA. ${ }^{15}$ The DNA was extracted as published previously. ${ }^{13}$

\section{Phenotype analysis}

Handedness was assessed through self-reporting using the Edinburgh Inventory questionnaire. ${ }^{16}$ Laterality quotient (LQ) scores were calculated by subtracting the number of tasks performed with the left hand from the number of tasks performed with the right hand. This number is then divided by the total number of tasks performed and multiplied by 100 . The LQ scores have a distribution from
-100 (complete left-handedness) to +100 (complete righthandedness). Families were only included in the genome scan analysis if LQ scores of $>50$ and $<-40$ were obtained for right-handed and left-handed individuals, respectively, and if their handedness for writing corresponded to the overall LQ score.

\section{Microsatellite analysis}

The genome-wide scan and genetic fine mapping were performed at the VIB Genetic Service Facility (http:// www.vibgeneticservicefacility.be/). Microsatellites were used from the ABI PRISM Linkage Mapping set MD-10 (Applied Biosystems, Foster City, USA). Multiplex PCRs were performed using the protocol from the Centre National de Génotypage (www.cng.fr). PCRs were performed with a True Allele Premix (Perkin-Elmer, Foster City, USA) containing $\mathrm{MgCl}_{2}$, AmpliTaq Gold, buffer and dNTPs on a Perkin-Elmer 9700 PCR engine. PCR conditions were provided by Perkin-Elmer and consisted of an initial denaturation step of $12 \mathrm{~min}$ at $94^{\circ} \mathrm{C}$ followed by 10 cycles consisting of a denaturation step $15 \mathrm{~s}$ at $94^{\circ} \mathrm{C}$, a $15 \mathrm{~s}$ annealing step at $55^{\circ} \mathrm{C}$ and an extension step at $72^{\circ} \mathrm{C}$ for $30 \mathrm{~s}$. These cycles were followed by 20 cycles with the same conditions except the denaturation step at $89^{\circ} \mathrm{C}$ and a final extension step at $72^{\circ} \mathrm{C}$ for $10 \mathrm{~min}$.

The PCR products were pooled together into 28 panels and were separated on a Perkin-Elmer 3700. Microsatellite analysis was performed using the program Genotyper (ABI Prism Perkin Elmer, Foster City, USA).

\section{Linkage analysis}

Parametric linkage calculations were performed using the programmes of the LINKAGE package. ${ }^{17}$ Simulation analysis was performed with the programmes SLINK $^{18}$ and SIMULATE, ${ }^{19}$ while linkage calculations were performed with the programme MLINK of the LINKAGE package. ${ }^{17}$ Linkage calculations were performed with equal allele frequencies. The model of Klar was coded as autosomal recessive with $50 \%$ penetrance, to reflect the fluctuating asymmetry component of the model, with gene frequencies of 58 and $42 \%$ for alleles $\mathrm{R}$ and $\mathrm{r}$, respectively. Nonparametric analysis was performed using the program GENEHUNTER. ${ }^{20}$

Simulation analysis with the programme SLINK was performed using 200 replications, while 1000 replications were used in the calculations with the programme SIMULATE. To test the results of the genome scan, calculations were performed using 5000 replications.

\section{Results \\ Phenotype analysis}

Handedness was assessed using the Edinburgh Inventory ${ }^{16}$ by calculating LQ scores, which indicate the direction of handedness (the hand subjects prefer to perform uni- 


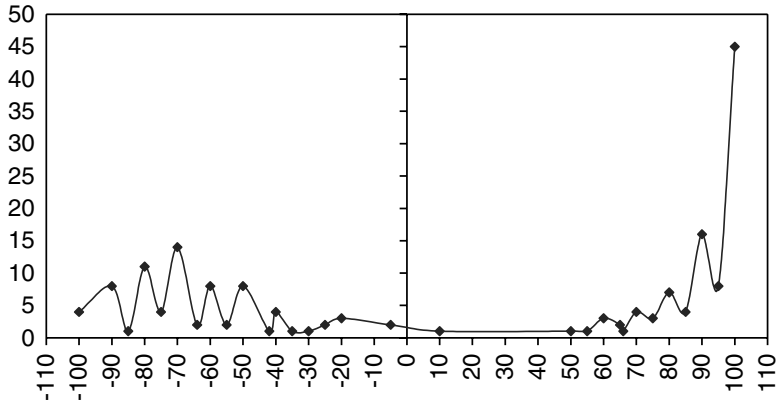

Figure 1 Distribution of the LQ scores. Scores lower than zero indicate left-handedness, while scores above zero indicate right-handedness.

manual tasks). In our sample cohort, the direction of handedness has a bimodal distribution, in which the majority of right-handed individuals have an LQ score of 100 and the majority of left-handed samples have an LQ score of -70 (see Figure 1). These results replicate previous results. ${ }^{16}$ A range of LQ scores can be observed for both lefthanded and right-handed individuals, with the left-handed individuals showing a greater range in LQ scores. This range in scores suggests that handedness is not a discrete variable. However, due to the bimodal distribution, handedness was treated as a qualitative phenotype.

\section{Linkage analysis}

\section{Simulation studies}

Parametric simulation analysis was performed to test the power of the collected sample cohort. Klar's model was coded for linkage analysis calculations as an autosomal recessive model with a reduced penetrance of $50 \%$ (see Materials and methods). To simulate a genome scan with an average marker heterozygosity of $75 \%$, simulation analysis using the model of Klar with 20 and 25 nuclear families was performed using allele frequencies of a marker with $70 \%$ heterozygosity. Using 25 families, a maximum LOD score of 7.4 was obtained with an average LOD score of 3.2. At a recombination fraction of 0.1 , an average maximum LOD score of 2.76 was obtained. With 200 replications, $57.5 \%$ of the replications reached a value higher than 3 .

Simulation analysis under the null hypothesis was performed with equal allele frequencies and 1000 replications. SIMULATE calculations with 20 nuclear families show that a maximum LOD score of 2.8 can be obtained by chance. An average maximum LOD score of 0.11 was obtained and 3\% of the replicates reached a value higher than 2 indicating that an observed LOD score of 2 has a pointwise $P$-value of less than 0.05 .

\section{Genome scan analysis}

Linkage analysis using Klar's model indicated the presence of four different regions in the genome that gave non- significant positive LOD scores with values higher than 1 . Two of these regions are located on chromosome 10 (Table 1). The first region is at position 10p12.33, where marker D10S197 resulted in an LOD score of 1.36 at a recombination fraction $(\theta)$ of 0.1 . The second region is at $10 q 26$ around marker D10S587 which gave an LOD score of 2.02 at $\theta=0.05$. In contrast to the region at $10 \mathrm{p} 12.33$, two flanking markers reached LOD values higher than 1 making the region at 10q26, a better candidate for harbouring a locus for human handedness. Nonparametric analysis using the programme GENEHUNTER resulted in an NPL score of 2.16 for marker D10S587 with a pointwise $P$-value of 0.02 .

The third and fourth regions are located on chromosomes 12 and 14, where D12S1659 and D14S276 gave a two-point LOD score of 1.86 and 1.26, respectively (Table 2). Nonparametric analysis of D12S1659 and D14S276 gave NPL scores of $1.82(P$-value $=0.04)$ and $1.67(P$-value $=0.05)$. However, two-point linkage analysis of the flanking markers in the $10 \mathrm{~cm}$ scan resulted in significant negative LOD scores in both the regions. In addition, the highest LOD scores were obtained at zero recombination fraction, which excludes the possibility of obtaining a more significant LOD score by analysing flanking markers.

The highest NPL scores were obtained for the regions discussed above and no other significant data were obtained using the programme GENEHUNTER.

Given the promising results of the region on chromosome 10q26, initial fine mapping was performed with three additional markers. D10S1483 is located $5 \mathrm{~cm}$ proximal to D10S587, between D10S587 and D10S1693, and has a maximum heterozygosity of 0.83. Markers D10S1656 and

Table 1 LOD score table of the markers surrounding the chromosome 10 regions that resulted in LOD scores above 1

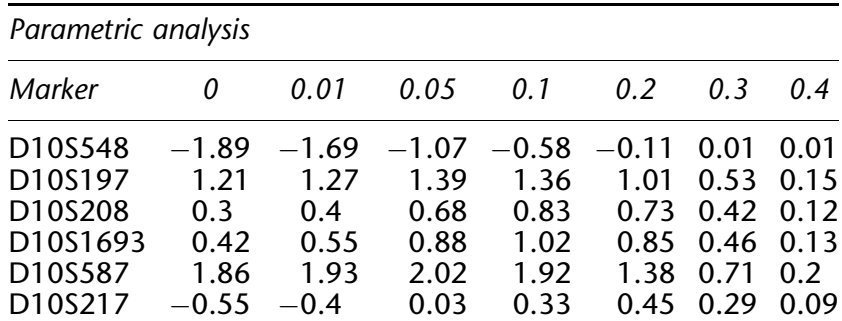

Nonparametric analysis

\begin{tabular}{lcc} 
Marker & NPL & P-value \\
\hline D10S548 & 0.17 & 0.44 \\
D10S197 & 1.72 & 0.04 \\
D10S208 & 1.44 & 0.07 \\
D10S1693 & 1.71 & 0.04 \\
D10S587 & 2.16 & 0.02 \\
D10S217 & 0.90 & 0.19
\end{tabular}

NPL stands for nonparametric LOD scores. 
Table 2 LOD score table of the chromosome 12 and chromosome 14 region

\begin{tabular}{lrrrrrrr}
\hline \multicolumn{7}{l}{ Parametric analysis } \\
\hline Marker & 0 & 0.01 & 0.05 & 0.1 & 0.2 & 0.3 & 0.4 \\
\hline D12S324 & -2.14 & -1.92 & -1.28 & -0.77 & -0.26 & -0.07 & -0.01 \\
D12S1659 & 1.86 & 1.85 & 1.76 & 1.56 & 1.03 & 0.51 & 0.14 \\
D12S1723 & -1.82 & -1.64 & -1.07 & -0.61 & -0.15 & -0.01 & 0.01 \\
D14S288 & -2.31 & -1.97 & -1 & -0.31 & 0.21 & 0.21 & 0.07 \\
D14S276 & 0.61 & 0.75 & 1.1 & 1.25 & 1.04 & 0.57 & 0.16 \\
D14S63 & -1.9 & -1.62 & -0.77 & -0.14 & 0.32 & 0.28 & 0.09 \\
Nonparametric analysis & & & & & \\
Marker & NPL & P-value & & & & & \\
\hline D12S324 & 0.01 & 0.50 & & & & & \\
D12S1659 & 1.82 & 0.04 & & & & & \\
D12S1723 & 0.19 & 0.41 & & & & & \\
D14S288 & 1.28 & 0.10 & & & & & \\
D14S276 & 1.67 & 0.05 & & & & & \\
D14S63 & 0.96 & 0.17 & & & & & \\
\hline
\end{tabular}

Table 3 Results of the fine mapping of the region on chromosome 10

\begin{tabular}{lrcccccl}
\hline Markers & 0 & 0.01 & 0.05 & 0.1 & 0.2 & 0.3 & 0.4 \\
\hline D10S1693 & 0.42 & 0.55 & 0.88 & 1.02 & 0.85 & 0.46 & 0.13 \\
D10S1483 & -0.02 & 0.15 & 0.64 & 0.92 & 0.88 & 0.51 & 0.15 \\
D10S587 & 1.86 & 1.93 & 2.02 & 1.92 & 1.38 & 0.71 & 0.2 \\
D10S1656 & -0.12 & -0.04 & 0.18 & 0.3 & 0.31 & 0.18 & 0.05 \\
D10S575 & -1.89 & -1.65 & -0.93 & -0.38 & 0.08 & 0.12 & 0.04 \\
D10S217 & -0.55 & -0.4 & 0.03 & 0.33 & 0.45 & 0.29 & 0.09
\end{tabular}

The markers of the genome scan are given in bold.

D10S575 are located 2 and $7 \mathrm{~cm}$, respectively, distal to D10S587 and have a maximum heterozygosity of 0.75 and 0.63 , respectively. However, all three markers resulted in negative LOD scores at zero recombination fraction (Table 3). Maximum positive LOD scores of 0.92 at $\theta=0.1$ and 0.31 at $\theta=0.2$ were obtained for markers D10S1483 and D10S1656, respectively.

\section{Discussion}

The genetics of human handedness is a highly debated topic and several models have been proposed to explain the complex epidemiology of left-handedness. Despite this, several genetic models propose that left-handedness is a monogenic trait with a non-Mendelian inheritance pattern and to our knowledge no genetic model has proposed that left-handedness is a true QTL. Consequently, we adopted a standard two-point parametric linkage analysis approach. Klar proposed a single-gene model that could explain and predict epidemiological data. ${ }^{10} \mathrm{Klar}^{\prime} \mathrm{s}$ model is the most reductionist model to our knowledge and hence can be readily used in linkage analysis studies.

It may be argued that the model of Klar, which was used in this project, is incorrect or incomplete, which may give spurious results. However, it has been suggested that twopoint analysis with a simple genetic model with reduced penetrance has the power to detect linkage for a trait with a complex mode of inheritance. ${ }^{21}$ The reduced penetrance makes the model robust enough to detect linkage and it mimics the effect of multiple loci. ${ }^{21}$ In addition, the high gene frequency specified in the model of Klar makes the model more robust as it allows for the parents passing on either haplotype if they are homozygous for the trait locus. ${ }^{22}$ It can therefore be concluded that although the model of Klar might not be correct, the characteristics of the model allow for it to be used in linkage calculations.

Four different regions with LOD scores higher than 1 have been identified. Chromosome 10q26 showed the most promise as a two-point LOD value of 2.02 was obtained, multiple markers reached positive LOD values and non-parametric analysis resulted in an NPL value of 2.16 ( $P$-value of 0.02 ). The obtained value is close to the value of 2.2, which represents a suggestive LOD score that may represent weak but real linkage. ${ }^{23}$ In addition, the highest LOD value was obtained at a recombination fraction of 0.05 , which indicates that upon further fine mapping a higher LOD value might be obtained. However, two-point analysis with markers situated at $5 \mathrm{~cm}$ from D10S587 resulted in lower LOD scores. These results can be explained by two possibilities.

The lower LOD scores obtained with the fine mapping might indicate a type I error (a false-positive result). However, it is also possible that only very weak linkage exists between a locus in the vicinity of D105587, rather than $5 \mathrm{~cm}$ away, and left-handedness in a subset of the analysed families. If the linkage only exists in a subset of the sample cohort, one would expect lower additive LOD scores due to the effect of the non-linked families. However, a much larger sample cohort will then be necessary to obtain sufficient power to identify loci for left-handedness.

Multipoint analysis is used routinely to increase the amount of power and LOD scores. However, using multipoint analysis when the exact genetic model is not known is problematic as this may give false-negative results due to the inflation of the recombination rate by the incorrect attribution of segregation of a disease allele. ${ }^{22}$ As two-point analysis is much more robust for misspecification than multipoint analysis, multipoint analysis was not employed.

Several reports have considered the involvement of the $\mathrm{X}$-chromosome in determining handedness. ${ }^{24,25}$ Association analysis between allele sharing of markers on the $\mathrm{X}$-chromosome and a reduction of hand skill variance in left-handed brothers resulted in an LOD score of 2.8 for marker DXS990. ${ }^{25}$ In this genome scan, no positive LOD scores were obtained for DXS990, thereby providing no evidence for the presence of a locus on the X-chromosome. QTL analysis using relative hand skill as the phenotype has 
identified a putative QTL on chromosome 2p11.2 between markers D2S2333 and D2S2216. ${ }^{26}$ Very recently this QTL was confirmed ${ }^{27}$ in a subset of left-handed male brothers from a previously collected sample cohort. ${ }^{25}$ However, we found no evidence of this QTL in this genome scan.

Given the amount of power present in our sample cohort, the characteristics of Klar's model, the NPL results and the robustness of two-point analysis against model misspecification, we can assume that if the monogenic models were correct, it would have been possible to detect this single locus. Hence, the genetic background of lefthandedness enables us to conclude that left-handedness is a true complex trait that is determined by the interaction of several different interacting loci. Further independent analysis using large sample cohorts is necessary to confirm the results of this study and to identify novel loci for human handedness.

\section{Acknowledgements}

We acknowledge Boris Harding, Els DeVriendt, Sam Sluijs and Hubert Backhovens of the VIB genetic Service Facility (http://www.vibgeneticservicefacility.be/) for their excellent assistance in the genetic analyses. In addition, we also thank all the families who participated in this study. The Murdoch Childrens Research Institute is a block-funded institute by the National Health and Medicine Research Council of Australia.

\section{References}

1 Gilbert AN, Wysocki CJ: Hand preference and age in the United States. Neuropsychologia 1992; 30: 601-608.

2 Coren S, Halpern DF: Left-handedness: a marker for decreased survival fitness. Psychol Bull 1991; 109: 90-106.

3 Knecht S, Drager B, Deppe M et al: Handedness and hemispheric language dominance in healthy humans. Brain 2000; 123: $2512-2518$.

4 Hicks RE, Kinsbourne M: Human handedness: a partial crossfostering study. Science 1976; 192: 908-910.

5 Carter-Saltzman L: Biological and sociocultural effects on handedness: comparison between biological and adoptive families. Science 1980; 209: 1263-1265.

6 McManus IC: The inheritance of left-handedness. Biological asymmetry and handedness. Ciba Found Symp 1991; 162: 251-281.

7 Sicotte NL, Woods RP, Mazziotta JC: Handedness in twins: a metaanalysis. Laterality 1999; 4: 265-286.
8 Annett M: Left, right, hand and brain; the right shift theory. Hove, UK Lawrence Erlbaum Associates Ltd 1985, pp 283-301.

9 McManus IC: Handedness, language dominance and aphasia: a genetic model. Psychol Med 1985, Monograph. Suppl. No 8.

10 Klar AJ: A single locus, RGHT, specifies preference for hand utilization in humans. Cold Spring Harb Symp Quant Biol 1996; 61: $59-65$.

11 McKeever WF: A new family handedness sample with findings consisitent with X-linked transmission. Br J Psychol 2000; 91 $21-39$.

12 Rife DC: Handedness, with special reference to twins. Genetics 1940; 25: 178-186.

13 Van Agtmael T, Forrest SM, Williamson R: Genes for lefthandedness: how to search for the needle in the haystack? Laterality 2001; 6: 149-164.

14 Van Agtmael T, Forrest SM, Williamson R: Parametric and nonparametric linkage analysis of several candidate regions for genes for human handedness. Eur J Hum Genet 2002; 10: 623-631.

15 Lench N, Stanier P, Williamson R: Simple non-invasive method to obtain DNA for gene analysis. Lancet 1988; 1: 1356-1358.

16 Oldfield RC: The assessment and analysis of handedness: the Edinburgh inventory. Neuropsychologia 1971; 9: 97-113.

17 Lathrop GM, Lalouel JM, Julier C, Ott J: Strategies for multilocus linkage analysis in humans. Proc Natl Acad Sci USA 1984; 81: $3443-3446$

18 Ott J: Computer-simulation methods in human linkage analysis. Proc Natl Acad Sci USA 1989; 86: 4175-4178.

19 Terwilliger JD, Speer M, Ott J: Chromosome-based method for rapid computer simulation in human genetic linkage analysis. Genet Epidemiol 1993; 10: 217-224

20 Kruglyak L, Daly MJ, Reeve-Daly MP, Lander ES: Parametric and nonparametric linkage analysis: a unified multipoint approach. Am J Hum Genet 1996; 58: 1347-1363.

21 Greenberg DA, Abreu P, Hodgem SE: The power to detect linkage in complex disease by means of simple LOD-score analyses. Am J Hum Genet 1998; 63: 870-879.

22 Risch N, Giuffra L: Model misspecification and multipoint linkage analysis. Hum Hered 1992; 42: 77-92.

23 Lander E, Kruglyak L: Genetic dissection of complex traits: guidelines for interpreting and reporting linkage results. Nat Genet 1995; 11: 241-247.

24 Corballis MC, Lee K, McManus IC, Crow TJ: Location of the handedness gene on the $\mathrm{X}$ and $\mathrm{Y}$ chromosomes. Am J Med Genet 1996; 67: 50-52.

25 Laval SH, Dann JC, Butler RJ et al: Evidence for linkage to psychosis and cerebral asymmetry (relative hand skill) on the $\mathrm{X}$ chromosome. Am J Med Genet 1998; 81: 420-427.

26 Francks C, Fisher SE, MacPhie IL et al: A genomewide linkage screen for relative hand skill in sibling pairs. Am J Hum Genet 2002; 70: 800-805.

27 Francks C, DeLisi LE, Fisher SE et al: Confirmatory evidence for linkage of relative hand skill to 2p12-q11. Am J Hum Genet 2003; 72: $499-502$. 